\title{
Repeat Accumulate Code Division Multiple Access and its Hybrid Detection
}

\author{
Rong Zhang, Lei Xu, Sheng Chen and Lajos Hanzo \\ School of ECS., Univ. of Southampton, SO17 1BJ, UK. \\ Tel: +44-23-80-593 125, Fax: +44-23-80-593045 \\ Email: lh@ecs.soton.ac.uk, http://www-mobile.ecs.soton.ac.uk
}

\begin{abstract}
In this paper, we propose the novel concept of Repeat Accumulate Code Division Multiple Access (RA-CDMA), which employs unique, user-specific channel code generator matrices (interleavers) for differentiating the users. We also suggest a Multilevel Structured (MLS) interleaver generation technique for the sake of reducing the memory storage requirements of our system and compare its correlation properties to those of the conventional random interleaver. A novel hybrid detector is designed for our system with the aid of EXtrinsic Information Transfer (EXIT) charts. Furthermore, we also design a low-complexity unequal power allocation scheme, assigning a different power to each user of this near-capacity transmission scheme. It is shown that when a sufficiently high interleaver length is employed, the RA-CDMA system is capable of approaching the Gaussian channel's capacity.
\end{abstract}

\section{INTRODUCTION}

A generalised Code Division Multiple Access (CDMA) technique may be defined as a multiple access scheme, which separates users in the 'generalised' code domain constituted by either classic spreading codes or by sophisticated channel codes, while sharing the same timeslots and frequency bands. In this generalised spirit, apart from the well-known Direct Sequence (DS) CDMA [1] scheme, two lesserknown generalised CDMA schemes were also proposed, namely Trellis Code Multiple Access (TCMA) [2] and Interleaver Division Multiple Access (IDMA) [3], both of which employ user-specific channel codes, represented by a unique combination of an interleaver and Trellis Coded Modulation (TCM) in TCMA and by 'stand-alone' unique interleavers in IDMA, respectively.

It was shown in [2] that in TCMA the employment of a unique user-specific interleaver after the TCM scheme is essential for the sake of attaining an attractive multi-user Bit Error Ratio (BER) performance. Thus TCMA may be viewed as a special case of IDMA, employing TCM codes as the outer channel code and removing the DS-spreading operation of IDMA, thus resulting in a potentially narrowband multiple access system. A specific feature of TCMA is that each user's transmitted symbol typically contains more than two bits. This potentially renders the receiver complex, when the number of users is high. High-complexity Maximum Likelihood (ML) detection was proposed in the original contribution on TCMA [2]. Another potential complexity-related issue is the high peakto-average power ratio of the transmitted signal having a large modulation signal constellation [4].

By contrast, IDMA has the benefit of employing low-complexity binary signalling for each user and yet, it is capable of achieving a high overall spectral efficiency. Naturally, increasing the number of users is achieved at the cost of sacrificing the individual users' throughput. Thus, IDMA is suitable for relatively low-rate UpLink (UL) communications. It was also shown in [3] that purely codespread IDMA but no spreading combined with appropriate power

Acknowledgments: The work reported in this paper has formed part of the Core 4 Research Programme of the Virtual Center of Excellence in Mobile and Personal Communications, Mobile VCE, www.mobilevce.com, whose funding support, including that of EPSRC, is gratefully acknowledged. Fully detailed technical reports on this research are available to Industrial Members of Mobile VCE. allocation is capable of approaching the channel capacity, with the aid of using Turbo-Hadamard codes for example [5]. The family Low Density Parity Check (LDPC) codes [6] becomes also attractive in the context of [7], since it has a built-in interleaver, which renders the employment of an extra user-specific interleaver after channel coding unnecessary.

Repeat Accumulate (RA) [8] codes having diverse coding rates also constitute attractive candidates for employment in code-spread IDMA. Similar to LDPC codes, RA codes also have an innate interleaver, while exhibiting a linearly increasing encoding complexity as a function of the codeword length. In this paper, we propose non-systematic regular RA Code Division Multiple Access (RACDMA), where different users are distinguished by their unique userspecific RA generator matrices, i.e. their interleavers. We design a Multilevel Structured (MLS) interleaver generation technique for the sake of reducing the interleaver's storage requirements and investigate its correlation properties compared to those of random interleavers. We also propose a novel hybrid detection scheme for the sake of supporting a high user-load in an equal user-power scenario. Furthermore, we discuss a low-complexity multi-user power-allocation method designed for our RA-CDMA system. It will be demonstrated that when a sufficiently high interleaver length is employed, the RA-CDMA system becomes capable of approaching the Gaussian channel's capacity. The contribution of this paper is that we propose a novel hybrid detection scheme for low-rate RA code-spread CDMA systems, as well as a MLS interleaver generation technique for the sake of reducing the interleaver storage requirements.

The rest of the paper is organised as follows. In Section II, we present the proposed RA-CDMA transceiver architecture. In Section III, we discuss several design aspects of our RA-CDMA system, namely the MLS interleaver generation technique advocated, the hybrid multiuser detector designed for equal-power allocation and finally, we suggest a low-complexity unequal power allocation scheme. We conclude our discourse in Section IV.

\section{System ARChitecture}

\section{A. Transmitter}

The $k$ th user's signal $b_{k}$ is encoded by its unique user-specific channel code $\mathcal{C}_{k}$ at a rate of $r$, generating the codeword $x_{k}=\mathcal{C}_{k}\left(b_{k}\right)$. In a traditional IDMA system, the channel code employed may be the same for all users and a unique user-specific interleaver is employed, which defines $x_{k}=\pi_{k}\left[\mathcal{C}\left(b_{k}\right)\right]$. The canonical discrete-time model of the multiple access channel seen in Fig. 1 is given by:

$$
y=\sum_{k=1}^{K} h_{k} \rho_{k} x_{k}+n,
$$

where $x \in\{ \pm 1\}, y$ and $n \sim \mathcal{N}\left(0, \sigma_{0}^{2}\right)$ denotes the transmitted signal, received signal and the Additive White Gaussian Noise (AWGN), respectively. Furthermore, $\rho_{k}$ contains the $k$ th user's amplitude subject to power allocation, while $h_{k}$ denotes the identical independently distributed UL Channel Impulse Response (CIR) of user $k$. RA codes 


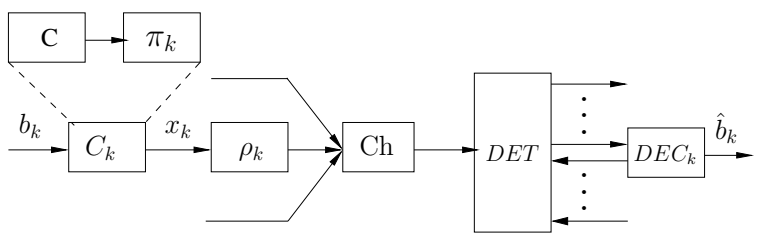

Fig. 1. Transceiver of the proposed RA-CDMA system

are employed as a benefit of their their low complexity, high codinggain and the availability of diverse code-rates. ${ }^{1}$

\section{B. Receiver}

An iterative receiver, consisting of a Soft-In Soft-Out (SISO) detector and a bank of $K$ individual SISO decoders is employed for the sake of seeking a tradeoff between the complexity of joint multiuser detection and decoding as well as the performance loss due to separate multiuser detection and single-user decoding. We introduce two detection algorithms, namely the optimal Maximum Likelihood (ML) detector and a low complexity Interference Cancellation (IC) aided detector, which can be implemented both as a Parallel IC (PIC) or as a Serial IC (SIC) scheme [3].

ML detector: The ML detector calculates the extrinsic information $L_{d e t}^{e}\left(x_{k}\right)$ based on the sum of all the $K$ users' $2^{K}$ conditional probabilities for the $l$ th legitimate noiseless output $\hat{y}_{l}$, given all other bits' a priori information $\phi$ corresponding to $x_{k}^{l}= \pm 1$, where $l \in 2^{K}$ :

$$
L_{d e c}^{e}\left(x_{k}\right)=\ln \frac{\sum_{\forall l: x_{k}^{l}=+1} \exp \left(-\frac{\left(\left\|y-\hat{y}_{l}\right\|\right)^{2}}{2 \sigma_{n}^{2}}+\phi\right)}{\sum_{\forall l: x_{k}^{l}=-1} \exp \left(-\frac{\left(\left\|y-\hat{y}_{l}\right\|\right)^{2}}{2 \sigma_{n}^{2}}+\phi\right)},
$$

where

$$
\phi=\frac{1}{2} \sum_{j \neq k} x_{j}^{l} L_{\text {det }}^{a}\left(x_{j}^{l}\right) .
$$

IC aided detector: Let us now rewrite (1) as

$$
y=H_{k} x_{k}+\xi,
$$

where $H_{k}=h_{k} \rho_{k}$ represents the composite CIR of user $k$, while $\xi=\sum_{j \neq k}^{K} H_{j} x_{j}+n$ represents the interference plus noise. In the case of binary modulation, the real part $(R e)$ of $H_{k}^{*} y$ constitutes sufficient statistics for estimating $x_{k}$, resulting in

$$
\operatorname{Re}\left(H_{k}^{*} y\right)=\left|H_{k}\right|^{2} x_{k}+\operatorname{Re}\left(H_{k}^{*} \xi\right)
$$

where $(\cdot)^{*}$ denotes the conjugate complex value of a variable. We denote the soft estimate of a variable $a$ by $(\hat{a})$. Then, $\operatorname{Re}\left(H_{k}^{*} \hat{\xi}\right)$ and its variance $\mathbf{V}\left[\operatorname{Re}\left(H_{k}^{*} \hat{\xi}\right)\right]$ are given by:

$$
\begin{aligned}
\operatorname{Re}\left(H_{k}^{*} \hat{\xi}\right)= & H_{k}^{R e} \hat{y}^{R e}+H_{k}^{I m} \hat{y}^{I m}-\left|H_{k}\right|^{2} \hat{x}_{k} \\
\mathbf{V}\left[\operatorname{Re}\left(H_{k}^{*} \hat{\xi}\right)\right]= & \left(H_{k}^{R e}\right)^{2} \mathbf{V}\left(\hat{y}^{R e}\right)+\left(H_{k}^{I m}\right)^{2} \mathbf{V}\left(\hat{y}^{I m}\right) \\
& -\left|H_{k}\right|^{4} \mathbf{V}\left(\hat{x}_{k}\right)+2 H_{k}^{R e} H_{k}^{I m} \phi,
\end{aligned}
$$

where $\phi=\sum_{k=1}^{K} H_{k}^{R e} H_{k}^{I m} \mathbf{V}\left(\hat{x}_{k}\right)$ and $\operatorname{Im}(\cdot)$ represents the imaginary part of a complex number. The soft estimate $\hat{y}$ and its variance can be expressed by:

$$
\begin{aligned}
\hat{y}^{R e} & =\sum_{k=1}^{K} H_{k}^{R e} \hat{x}_{k}, \\
\mathbf{V}\left(\hat{y}^{R e}\right) & =\sum_{k=1}^{K}\left(H_{k}^{R e}\right)^{2} \mathbf{V}\left(\hat{x}_{k}\right)+\sigma_{n}^{2} .
\end{aligned}
$$

\footnotetext{
${ }^{1}$ The encoding and decoding procedure of RA codes [8] are excluded from this paper due to space limitations.
}

We remark that (8) and (9) would be equally valid for the imaginary counterpart. The soft bit $\hat{x}_{k}$ can be represented as $\hat{x}_{k}=$ $\tanh \left(L_{d e c}^{e}\left(x_{k}\right) / 2\right)$, while its variance is given by $\mathbf{V}\left(\hat{x}_{k}\right)=1-$ $\hat{x}_{k}^{2}$. Assuming $\xi$ is Gaussian distributed, the extrinsic information $L_{\text {det }}^{e}\left(x_{k}\right)$ is given by:

$$
L_{\text {det }}^{e}\left(x_{k}\right)=2\left|H_{k}\right|^{2} \frac{\operatorname{Re}\left(H_{k}^{*} y\right)-\operatorname{Re}\left(H_{k}^{*} \hat{\xi}\right)}{\mathbf{V}\left[\operatorname{Re}\left(H_{k}^{*} \hat{\xi}\right)\right]} .
$$

Then the extrinsic information forwarded from the detector is used as a priori information of the RA decoder, which in turn generates more reliable extrinsic information $L_{d e c}^{e}\left(x_{k}\right)$ for the next iteration.

\section{DESIGN OF RA-CDMA}

\section{A. Interleaver Generation Technique}

We consider non-systematic RA codes in this paper, which have a repetition depth of $L$ and information packets length of $Q$. Similar to LDPC codes, the PCM of an RA code can be represented by $\mathbf{H}=\left[\mathbf{H}_{1}, \mathbf{H}_{2}\right]$, where $\mathbf{H}_{1}$ is a $(Q L \times Q)$-element matrix specified by the interleaver and $\mathbf{H}_{2}$ is a $(Q L \times Q L)$-element matrix specified by the accumulator:

$$
\mathbf{H}_{2}=\left[\begin{array}{llllll}
1 & 0 & 0 & & 0 & 0 \\
1 & 1 & 0 & & 0 & 0 \\
0 & 1 & 1 & & 0 & 0 \\
& & & \ddots & & \\
0 & 0 & 0 & & 1 & 0 \\
0 & 0 & 0 & & 1 & 1
\end{array}\right] .
$$

The user-specific RA codes become unique by appropriately designing their inherent user-specific interleavers $\pi_{k}^{u}$. This implies that a different Parity Check Matrix (PCM) must be stored in a memoryinefficient Look-Up Table (LUT) for each user in the context of multiuser. Thus the memory storage requirements are dependent on both the codeword length, the PCM parameters such as the column (or row) weight as well as the number of users in the system. Inspired by the efficient Nested interleaver concept of [9], here we propose novel user-specific MLS interleavers.

1) Construction of MLS Interleavers: The proposed MLS interleavers are constructed from a system-specific base interleaver, a userspecific base interleaver, a so-called constituent interleaver set, which are then subjected to a position sorting operation, all of which are detailed below.

The system-specific base interleaver $\pi^{b}$ is a randomly generated interleaver of length- $Q$. Each user has a distinct user-specific base interleaver $\pi_{k}^{b}, k=1, \ldots, K$ having the same length- $Q$ as the system-specific base interleaver $\pi^{b}$. The $(k+1)$ st user-specific base interleaver is an interleaved version of the $k$ th user-specific base interleaver rearranged by the system-specific base interleaver $\pi^{b}$, as follows: $\pi_{k+1}^{b}=\pi^{b}\left(\pi_{k}^{b}\right)$ and $\pi_{1}^{b}=\pi^{b}$.

The constituent interleaver set of user $k$ is represented by $L$ number / level of length- $Q$ interleavers, which is formulated as $\pi_{k}^{s}=\left\{\pi_{1}, \pi_{2}, \ldots, \pi_{L}\right\}$. Each element $\pi_{l} \in \pi_{k}^{s}, l=1, \ldots, L$ of the constituent interleaver set is a distinct length- $Q$ interleaver, having the same length as the system-specific base interleaver $\pi^{b}$. The $(l+1)$ st constituent interleaver is an interleaved version of the $l$ th constituent interleaver rearranged by the user-specific base interleaver $\pi_{k}^{b}$, according to $\pi_{l+1}=\pi_{k}^{b}\left(\pi_{l}\right)$ and $\pi_{1}=\pi_{k}^{b}$.

Finally, the $L$ number of length- $Q$ interleavers are concatenated to form a unique length- $L Q$ interleaver. This is carried out by the constituent interleaver set position sorting operation, as defined by the position mapping function $f$, which maps the index $q_{l}=1, \ldots, Q$ within all the $L$ number of length- $Q$ constituent interleavers $\pi_{l} \in$ $\pi_{k}^{s}, l=1, \ldots, L$ into a single user-specific interleaver $\pi_{k}^{u}=f\left(\pi_{k}^{s}\right)$ 
unambiguously mapping the $L Q$ number of input bit positions to the interleaved positions $q=1, \ldots, L Q$. More specifically, the index $q_{l}=1, \ldots, Q$ within any of the $L$ number of length- $Q$ constituent interleaver $\pi_{l}, l=1, \ldots, L$ is mapped to $q=\left(q_{l}-1\right) L+l$.

2) Cross-correlation Evaluation: Let us now demonstrate the equivalence of our proposed MLS interleavers to random interleavers in terms of the correlation metric introduced in [9]. In other words, our goal is to demonstrate that despite its significantly reduced memory requirements, the proposed interleaver generation technique does not increase the correlation between the pairs of interleaved information sequences in comparison to using random interleavers.

The correlation $\chi$ between two independently generated random information bit sequences $s_{1}$ and $s_{2}$ interleaved by two different interleavers $\pi_{1}$ and $\pi_{2}$ is given by the scalar product $\circ$ between $\pi_{1}\left(s_{1}\right)$ and $\pi_{2}\left(s_{2}\right)$, which can be written as:

$$
\chi=\left|\pi_{1}\left(s_{1}\right) \circ \pi_{2}\left(s_{2}\right)\right| .
$$

Since evaluating the correlation amongst all possible pairs of random sequences $s_{1}$ and $s_{2}$ has a high computational cost, we seek a lowercomplexity alternative [9]. We represent $s_{1}$ as $s_{1}=\sum_{i=1}^{N} \alpha_{i} \mathbf{b}_{i}$, where $\alpha_{i} \in\{ \pm 1\}$ and the vector $\left\{\mathbf{b}_{i}: \mathbf{b}_{i}(i)=1, \mathbf{b}_{i}(j)=0\right\}$ of length $N$ is a vector within the basis set $\mathbf{B}=\left[\mathbf{b}_{1}, \mathbf{b}_{2}, \ldots, \mathbf{b}_{N}\right] \subset$ $\mathbb{R}^{N}$. On the other hand, $s_{2}$ can be replaced by generating a set $\mathbf{G}=$ $\left[\mathbf{g}_{1}, \mathbf{g}_{2}, \ldots, \mathbf{g}_{N}\right] \subset \mathbb{R}^{N}$, where each vector $\mathbf{g}_{i}$ of length $N$ has an entry of $\mathbf{g}_{i}(j)=-1$ when we have $j<i$ and $\mathbf{g}_{i}(j)=1$ for $j \geq i$. Thus the correlation of Eq. (11) becomes the so-called upper-bounded basis correlation vector $\chi^{b}=\left[\chi_{1}^{b}, \chi_{2}^{b}, \ldots, \chi_{N}^{b}\right]$ defined in [9], where each entry $\chi_{j}^{b}, j=1, \ldots, N$ is represented as:

$$
\chi_{j}^{b}=\sum_{i=1}^{N}\left|\pi_{1}\left(\mathbf{b}_{i}\right) \circ \pi_{2}\left(\mathbf{g}_{j}\right)\right| .
$$

Fig. 2 demonstrates the normalised average histogram of the correlations recorded for both random interleavers and for our proposed MLS interleavers. The total interleaver length was set to $N=1024$ and we divided them into $L=4$ constituent interleavers, each having a length of $Q=256$. We averaged the correlations over both 100 pairs of random interleavers and 100 pairs of our proposed MLS interleavers. Fig. 2 suggests that statistically speaking, both interleaver families exhibit similar correlations.

Furthermore, the system-specific base interleaver $\pi^{b}$ can be generated by a shift register based $m$-sequence generator. Thus, our proposed scheme, which exhibits similar correlation properties to those of random interleavers, does not require the storage of all the $K$-users' interleavers. Only $m$ bits have to be stored, where $m$ in our system is related to the length $Q=2^{m}$ of the source information packets, rather than to the length $L Q$ of the RA-CDMA coded packets. This is lower than the storage requirements of the basic Nest interleavers proposed in [9], especially when the RACDMA coded packets are long.

\section{TABLE I}

SUMMARY OF THE SIMULATION PARAMETERS.

\begin{tabular}{l||c}
\hline \hline Modulation Type & BPSK \\
\hline Channel Type & AWGN (Fig.3) and UR (Fig.5) \\
\hline Rate of RA Code & 0.25 \\
No. of MLS levels & 4 \\
Info. Packet Length $(Q)$ & 1024 \\
\hline Iter No. (Det and Dec) & 5 (4 users), 10 (6 users), 20 (8 users) \\
Iter No. (SPA) & 20 \\
\hline
\end{tabular}

3) Simulation results: Fig. 3 compared the BER performance of our proposed MLS RA-CDMA scheme, where the user-separation is
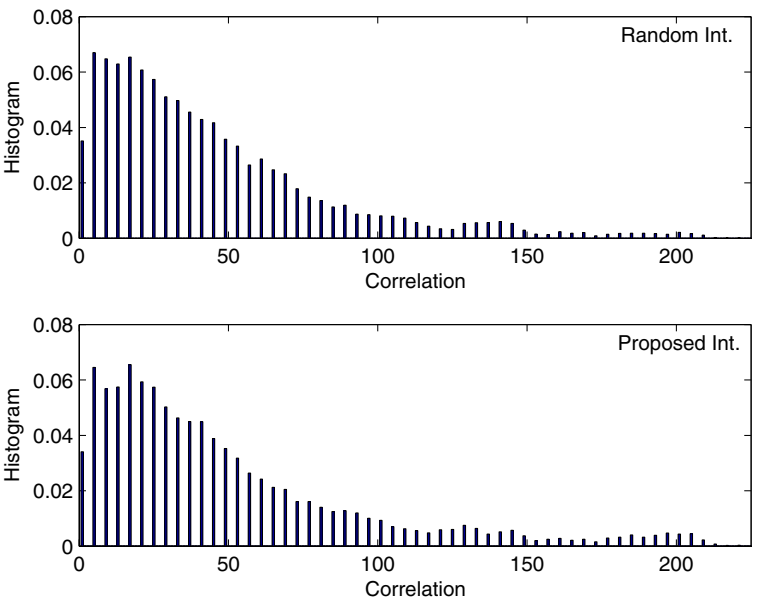

Fig. 2. The normalisd average histogram of the correlation for both random interleavers and our proposed MLS interleavers. The total interleaver length was set to $N=1024$ and we divided them into $L=4$ constituent interleavers, each having length of $Q=256$. A total number of 100 pairs of interleavers of both random interleavers and our proposed MLS interleavers were averaged for generating the results.

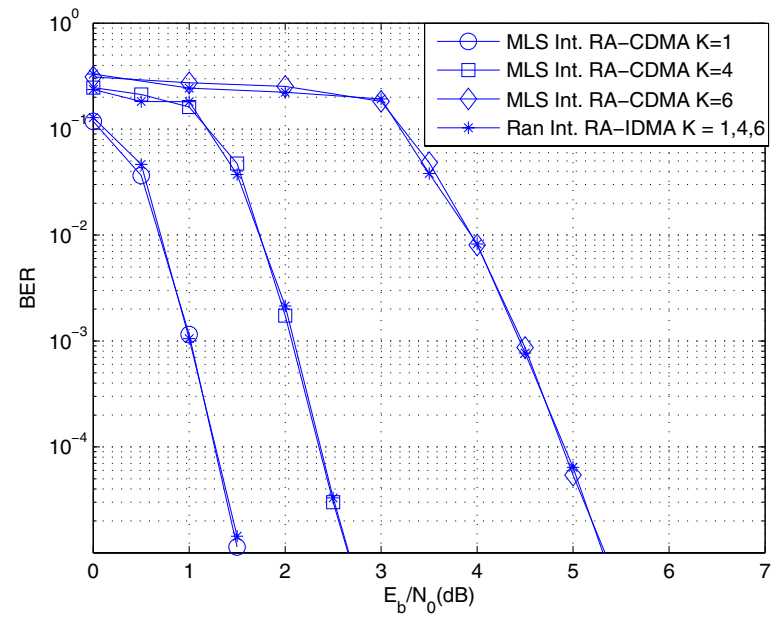

Fig. 3. Comparison of the BER performance of our proposed MLS RA-CDMA to that of conventional randomly generated RA coded IDMA configuration. The simulation parameters are given in Table I.

achieved by user-specific MLS interleavers to that of a conventional randomly generated RA coded IDMA configuration [3], where the user separation is achieved by user-specific interleavers after channel coding. The simulation parameters are summarized in Table I. Fig. 3 shows that our proposed system does not suffer from any BER performance loss, which implies that having an extra interleaver after channel coding is redundant in conventional RA coded IDMA systems and hence the employment of 'stand-alone' unique userspecific MLS generator matrices is sufficient in our RA-CDMA system.

\section{B. Hybrid Detection}

1) EXIT chart analysis: Let us firstly investigate the convergence behaviour of the iterative multiuser receiver in an equal user-power scenario, i.e. when we have $\rho_{k}=\rho, \forall k$ using EXtrinsic Information Transfer (EXIT) charts [10]. Fig. 4 compares two detector's EXIT curves for a $K=8$-user RA-CDMA system communicating over an 


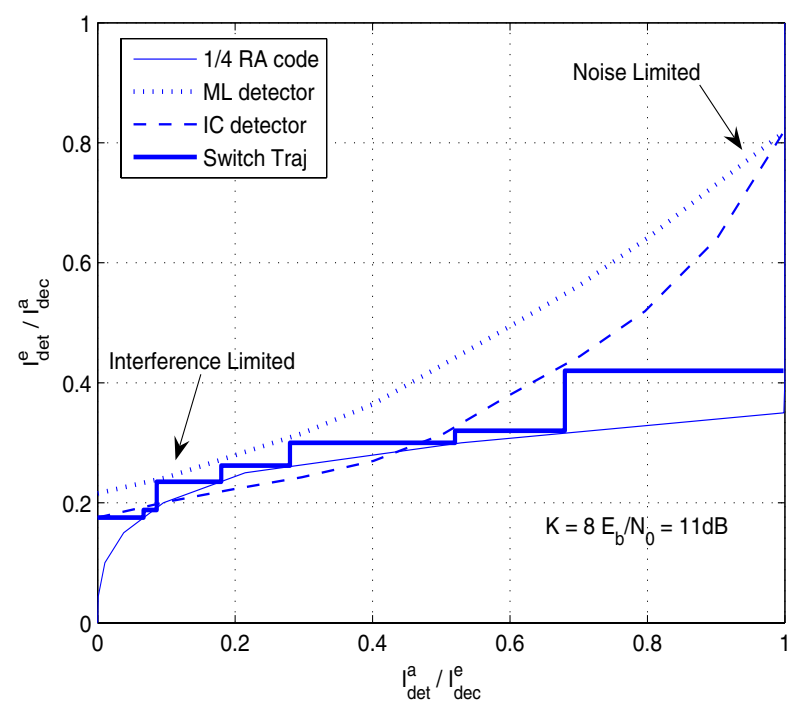

Fig. 4. EXIT chart analysis of the RA-CDMA system.

uncorrelated non-dispersive Rayleigh fading channel, when employing a code-rate of $r=1 / 4$ at $E_{b} / N_{0}=11 d B$, where we employed a sub-optimum PIC detector and the optimum ML detector.

At the left of Fig. 4, which corresponds to the interference-limited region, the ML detector outputs only marginally higher extrinsic information than the PIC detector. As the amount of available $a$ priori information increases, the discrepancy between these two EXIT curves becomes more substantial owing to the suboptimal nature of the PIC detector. Ultimately, these two curves tend to the corresponding single-user performance, when the effects of interference have been more or less eliminated, corresponding to the noise-limited region at the right of Fig. 4.

2) Hybrid detector: As seen in Fig. 4, the PIC detector has a more limited ability to exploit the extrinsic information during the consecutive iterations compared to the ML detector and hence its performance is suboptimum. Our novel proposition is to use the PIC detector during the first few iterations and then subsequently activate the higher-complexity, but more powerful ML detector for a few further iterations to avoid encountering the "bottleneck" region in the EXIT chart of the PIC detector. Beyond the EXIT-chart's bottleneck region we may then safely activate again the lower-complexity PIC detector.

In order to determine the activation instant of the different detectors, the average of the extrinsic information's absolute value $\left|L_{d e t}^{e}\right|$ generated by the detector is invoked, which is supposed to be monotonically increasing as the number of iterations is increased. The value of $\left|L_{d e t}^{e}\right|$ is recorded after each detector iteration and then compared to that of the previous iteration, resulting in the incremental value $\Delta$ as:

$$
\Delta=\left|L_{\text {det }}^{e, l}\right|-\left|L_{\text {det }}^{e, l-1}\right| .
$$

More explicitly, we determine the detector activation order as follows:

Step 1: Initially, we activate the PIC detector.

Step 2: At iteration $l$, if $\Delta<\Delta_{t}$, the ML detector is activated, where $\Delta_{t}$ is a small experimentally predefined positive value.

Step 3: At iteration $l$, we determine:

1) the actual extrinsic mutual information value $I_{d e c}^{e, l}$ from the decoder based on the recorded $\left|L_{d e t}^{e, l}\right|$.

2) the required a priori mutual information $I_{d e t}^{a, l}$ for the PIC

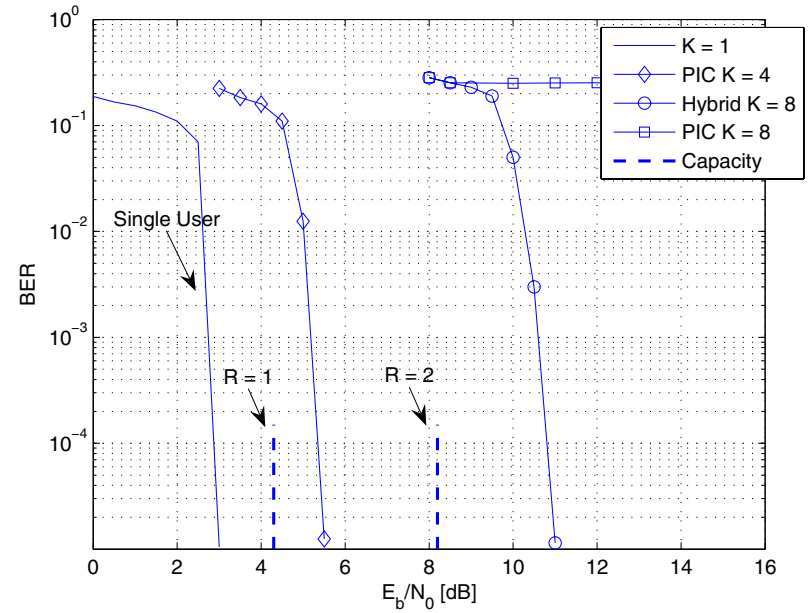

Fig. 5. Performance of the rate $r=1 / 4$ RA-CDMA system for transmission over a non-dispersive, uncorrelated Rayleigh fading channel using the proposed hybrid detector. The simulation parameters are given in Table I.

detector to get the same $\left|L_{d e t}^{e, l}\right|$.

If $I_{d e c}^{e, l}-I_{d e t}^{a, l}>\delta$, where $\delta$ is a small positive predefined value, we switch to the PIC detector.

As seen in Fig.4, the simulation-based stair-case-shaped detection trajectory closely follows the EXIT curves of the receiver components employing our algorithm. During the initial few iterations, the PIC detector is activated. As the incremental iteration gain of each additional iteration is reduced, the ML detector becomes activated, in order to overcome the PIC detector's EXIT tunnel constriction. When the consecutive iteration gains of the ML detector become sufficiently high again, the PIC detector is reactivated in the interest of reducing the complexity imposed. In order to avoid any potential failure to converge, the threshold of $\delta$ used for switching to the PIC detector should be appropriately adjusted.

As an explicit benefit, the proposed hybrid detector becomes capable of achieving a similar performance to that of the potentially excessive-complexity ML detector at a moderate complexity, since it is only activated during the critical detector iterations. Moreover, the ML detector can be replaced by any low-complexity near-ML type detector, such as a Sphere Detector (SD) [4], or a Genetic Algorithm (GA) aided detector [4], in order to further reduce the overall complexity.

3) Simulation results: Fig.5 portrays the attainable BER performance of both the PIC and of the proposed hybrid detectors as a function of the $E_{b} / N_{0}$ and as well as of the number of users supported. The simulation parameters are given in Table I. Observe in Fig. 5 that at a multiuser sum-rate of $R=K \times r=2$, the PIC detector fails to converge, while the proposed hybrid detector achieves an infinitesimally low BER at $E_{b} / N_{0} \approx 11 d B$. This $E_{b} / N_{0}$ value is only about $3 \mathrm{~dB}$ away from the ergodic Rayleigh fading channel's capacity [11].

\section{Power Allocation}

The capacity of the scalar Gaussian multiple access channel can be approached by SIC and decoding combined with optimum power- or rate-allocation [12]. Motivated by this, we propose a low-complexity power allocation scheme for our system employing binary nearcapacity RA codes, which are capable of approaching the capacity for sufficiently long information block lengths, given a total multiuser power constraint of $P=\sum_{k=1}^{K} P_{k}$. We assume having an equal 


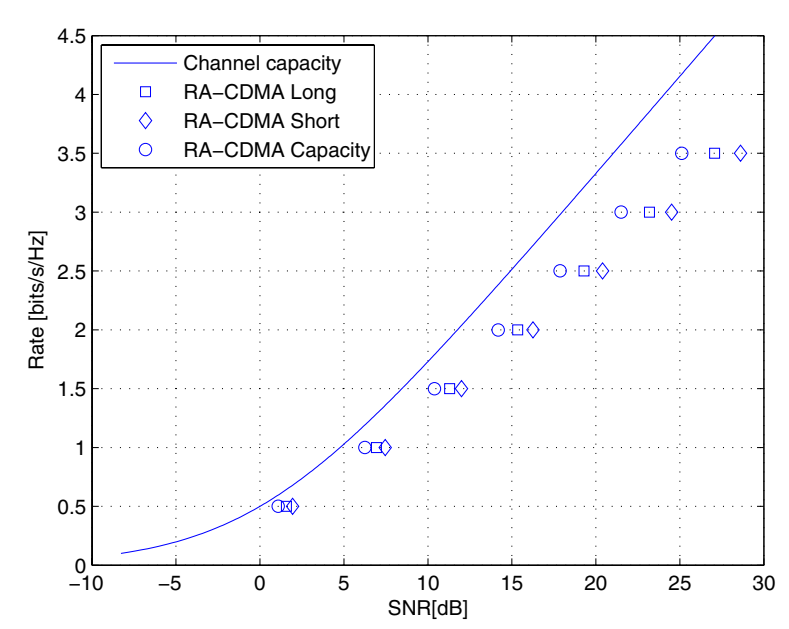

Fig. 6. The achievable rate of RA-CDMA using the proposed low-complexity power allocation scheme for both a short information sequence length of 4096 and long information sequence length of 10000 bits.

user-rate scenario, i.e. that we have $R=K r$, where $R$ and $r$ denotes the sum of the user rates and each user's rate, respectively.

The minimum required Signal to Noise Ratio (SNR) $\mathrm{SNR}^{m}$ at a given multiuser sum-rate $R$ is given by [12], $\mathrm{SNR}^{m}=\left(2^{2 R}-1\right)$. Our proposed power allocation scheme operates as follows. We set a small incremental value $\tau$ for representing the difference between the minimum $\mathrm{SNR}^{m}$ and the required $\mathrm{SNR}^{r}$ at the multiuser sum-rate $R$ as follows:

$$
\tau=\left(\mathrm{SNR}^{r}\right)_{d B}-\left(\mathrm{SNR}^{m}\right)_{d B}
$$

According to the individual RA decoder of user $k$, we have a threshold value $\mathrm{SNR}^{\mathrm{t}}$, at which the RA decoder exhibits an infinitesimally low BER. This threshold may be acquired by simulations or by using a semi-analytic EXIT chart based prediction. Then the power allocated to user $k$ is given by:

$$
\hat{P}_{k}=\operatorname{SNR}^{t}\left(\sum_{j=k+1}^{K} \hat{P}_{j}+\frac{P}{10^{\frac{\tau}{10}} \mathrm{SNR}^{m}}\right) .
$$

Furthermore, the difference between the minimum SNR and the required SNR of user $k$ is given by:

$$
\tau_{k}=\left(2^{2 r}-1\right)_{d B}-\left(\mathrm{SNR}^{t}\right)_{d B} .
$$

Following the aforementioned power allocation procedure, we calculate the total power consumption according to $\hat{P}=\sum_{k=1}^{K} \hat{P}_{k}$. If we have $\hat{P}>P$, we set a larger incremental value $\tau$ and reallocate the individual users' power. The power allocation is achieved when we have $\hat{P}=P$ and the resultant threshold value $\tau$ quantifies the distance from capacity for the multiuser sum-rate $R$.

Fig 6 shows the achievable rate of the RA-CDMA system using the proposed power allocation regime for both a short information sequence length of 4096 and for a long information sequence length of 10000 bits. Although not explicitly shown here due to space limitations, when a rate $r=1 / 4$ RA code was employed, the actual $E_{b} / N_{0}$ values required at these two information sequence lengths for achieving an infinitesimally low BER were observed to be at $0.8 \mathrm{~dB}$ and $0.5 \mathrm{~dB}$, when using either bit-by-bit simulation or EXIT chart predictions, respectively, in conjunction with $I_{R A}=50$ RA decoding iterations. The minimum decoding threshold $E_{b} / N_{0}$ value for a rate $r=1 / 4 \mathrm{RA}$ code having an infinite information sequence length was reported to be $0.1 \mathrm{~dB}$ when using the sum-product decoding algorithm [8]. Hence we may refer to the corresponding achievable rate as the approximate RA-CDMA capacity.

Observe in Fig 6 that RA-CDMA system is capable of approaching the Gaussian channel's capacity, when employing the proposed power allocation scheme. When $\tau_{k}$ is small, the overall discrepancy $\tau$ of Eq. (14) will also be small and as a benefit of the power allocation algorithm contrived, the system operates close to the achievable channel capacity, as seen in Fig. 6. However, the overall difference $\tau$ formulated in Eq. (14) increases further, when the number of users $K$ becomes high, especially when a short information sequence length is used. This is because our power allocation scheme does not take into account the iterative receiver's operation, which results in a small $\tau$ at a high multiuser sum-rate. This drawback can be eliminated by the simulation based power allocation scheme of [13], which does take into account the iterative receiver's operation at the cost of a higher complexity. Thus our low-complexity power allocation is suitable in moderate multiuser sum-rate scenario, e.g. $R \leq 3$.

\section{CONCLUSION}

In this paper, we proposed a novel RA-CDMA transceiver architecture, which may be viewed as a low-complexity IDMA scheme employing a new MLS interleaver generation technique. It was also demonstrated that our system is capable of supporting a high multiuser sum-rate with the aid of the proposed hybrid detectors under the assumption of having an equal power scenario. Furthermore, the system is capable of approaching the Gaussian channel's capacity with the aid of the low-complexity unequal power allocation scheme proposed. In our future work, various practical power allocation schemes and interleaver acquisition schemes will be investigated.

\section{REFERENCES}

[1] L. Hanzo, L.-L. Yang, E.-L. Kuan, and K. Yen, Single- and Multi-Carrier DS-CDMA: Multi-User Detection, Space-Time Spreading, Synchronisation, Networking and Standards. Wiley-IEEE Press, 2003.

[2] F. Brannstrom, T. Aulin, and L. Rasmussen, "Iterative detectors for trellis-code multiple-access," IEEE Transactions on Communications, vol. 50, pp. 1478-1485, Sept. 2002.

[3] L. Ping, L. Liu, K. Wu, and L. W. K., "Interleave-division multipleaccess," IEEE Transactions on Wireless Communications, vol. 5, pp. 938-947, Apr. 2006.

[4] L. Hanzo and T. Keller, OFDM and MC-CDMA: A Primer. Wiley-IEEE Press, 2006.

[5] L. Ping, W. K. Leung, and K. Y. Wu, "Low-rate turbo-Hadamard codes," IEEE Transactions on Information Theory, vol. 49, pp. 3213-3224, Dec. 2003.

[6] R. G. Gallager, Low-Density Parity-Check Codes. MIT Press, Cambridge, 1963 .

[7] N. Varnica, A. Kavcic, X. Ma, and L. Ping, "Density evolution and LDPC code optimization for interleaver division multiple accesss," in Proc. of IEEE GMC 04', Shanghai, China, May11-13, 2004, pp. 381385.

[8] H.Jin, Analysis and Design of Turbo-like Codes. Ph.D. Thesis, California Institute of Technology, Pasadena, 2001.

[9] I. Pupeza, A. Kavcic, and L. Ping, "Efficient generation of interleavers for IDMA," in Proc. of IEEE ICC 06', Istanbul, Turkey, June 11-15, 2006.

[10] S. ten Brink, "Convergence behavior of iteratively decoded parallel concatenated codes," IEEE Transactions on Communications, vol. 49, pp. 1727-1737, Oct. 2001.

[11] D. Tse and P. Viswanath, Fundamentals of Wireless Communication. Cambridge University Press, 2005.

[12] T. Cover and J. Thomas, Elements of Information Theory. New York: Wiley, 1991.

[13] L. Liu, J. Tong, and L. Ping, "Analysis and optimization of CDMA systems with chip-level interleavers," IEEE Journal on Selected Areas in Communication, vol. 24, pp. 141 - 150, Jan. 2006. 